Pacific Journal of Mathematics

BETWEENNESS RELATIONS IN PROBABILISTIC METRIC 


\title{
BETWEENNESS RELATIONS IN PROBABILISTIC METRIC SPACES
}

\author{
R. Moynihan AND B. Schweizer
}

\begin{abstract}
Four distinct versions of the betweenness concept for probabilistic metric spaces are defined and studied. Conditions under which some or all of the properties of metric betweenness are satisfied are determined and the relationships among the different concepts are investigated.
\end{abstract}

1. Introduction. In his original paper on probabilistic metric spaces [8] K. Menger, in addition to introducing the basic concepts and axioms, introduced a definition of betweenness, developed some of its properties and showed that this relation was generally weaker than ordinary metric betweenness. Shortly thereafter, A. Wald [25] introduced a different definition of betweenness, based on a different triangle inequality, and showed that his relation did have all the properties of metric betweenness. Subsequently, J. F. C. Kingman [6] and F. Rhodes [16] studied betweenness in "Wald spaces" and $H$. Sherwood [23] considered a probabilistic version of the concept. Otherwise the subject has lain dormant-primarily because adequate tools for its analysis were not available. Our recent work on the structure of semigroups on the space of probability distribution functions $[9,11,12,17]$ and the development of "characteristic functions" for certain classes of these semigroups $[10,13,14]$ has changed this state of affairs. Thus we return to the study of betweenness in probabilistic metric spaces. We focus our attention on four different versions of this concept. The first of these is the straightforward generalization of Wald's betweenness from Wald spaces to arbitrary probabilistic metric spaces. We show that this relation satisfies some, but generally not all, of the usual properties of metric betweenness, determine sufficient conditions for the validity of those properties which are not always satisfied and show that in some instances these conditions are also necessary. The second betweenness relation applies to a restricted but nevertheless very large class of probabilistic metric spaces. It always satisfies the metric betweenness properties and, whenever it is comparable to the first relation, it is either identical or weaker. The third relation, which applies to the same class of spaces as the second, is obtained by an extension of Kingman's idea from Wald spaces to this class. It is a metric betweenness for certain naturally defined metrics and is always weaker than the second relation. The last relation is Menger's betweenness. We reformulate Menger's definition in terms of triangle functions and show that in 
simple spaces and Wald spaces, Wald's and Menger's concepts coincide. A more detailed study of Menger's betweenness still remains to be carried out.

In order to present our results we need to recapitulate some of the basic definitions and known results from the theory of probabilistic metric (PM) spaces. Recall that such a space is an ordered triple $(S, \mathscr{F}, \tau)$, where $S$ is a set, $\tau$ is a triangle function, and $\mathscr{F}$ is a mapping from $S \times S$ into the set of distribution functions

$$
\begin{aligned}
\Delta^{+}=\{F: R \rightarrow & {[0,1] \mid F \text { is nondecreasing, left-continuous } } \\
& \text { and } F(0)=0\}
\end{aligned}
$$

such that, for all $p, q, r$ in $S$,

(I) $F_{p q}=\varepsilon_{0}$ if and only if $p=q$,

(II) $F_{p q}=F_{q p}$,

(III) $\quad F_{p r} \geqq \tau\left(F_{p q}, F_{q r}\right)$.

Here $F_{p q}=\mathscr{F}(p, q) ; \varepsilon_{0}$ is the distribution function defined by

$$
\varepsilon_{0}(x)= \begin{cases}0, & x \leqq 0 \\ 1, & 0<x ;\end{cases}
$$

and a triangle function $\tau$ is a binary operation on $\Delta^{+}$satisfying

( $\alpha) \tau\left(F, \varepsilon_{0}\right)=F$,

( $\beta) \tau\left(F_{1}, G_{1}\right) \geqq \tau\left(F_{2}, G_{2}\right)$, whenever $F_{1} \geqq F_{2}, G_{1} \geqq G_{2}$,

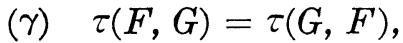

(ס) $\tau(\tau(F, G), H)=\tau(F, \tau(G, H))$.

Thus $\tau$ is a commutative, order-preserving semigroup operation, with unit $\varepsilon_{0}$, on $\Delta^{+}$.

A sequence $\left\{F_{n}\right\}$ in $\Delta^{+}$converges weakly to $F \in \Delta^{+}$, and we write $F_{n} \stackrel{w}{\rightarrow} F$, if and only if the sequence $\left\{F_{n}(x)\right\}$ converges to $F(x)$ at every continuity point $x$ of the limit function $F$. This mode of convergence is metrizable (an explicit metric is exhibited in [24]) and the space $\Delta^{+}$is compact in the induced metric topology. If the triangle function $\tau$ is (uniformly) continuous then the collection of $\operatorname{sets}\left\{N_{p}(\varepsilon, \lambda) \mid \varepsilon>0, \lambda>0, p \in S\right\}$, where

$$
N_{p}(\varepsilon, \lambda)=\left\{q \in S \mid F_{q p}(\varepsilon)>1-\lambda\right\}
$$

is a neighborhood basis for a metrizable topology on $S[18,21]$, called the $\varepsilon, \lambda$-topology. Moreover, a sequence $\left\{q_{n}\right\}$ in $S$ converges to $q \in S$ in this topology if and only if $F_{q q_{n}} \stackrel{w}{\rightarrow} \varepsilon_{0}$.

When $\tau$ is convolution (a continuous triangle function), then $(S, \mathscr{F})$ is a Wald space; and $(S, \mathscr{F})$ is a Menger space when $\tau$ is ${ }_{3}$ of the form $\tau_{T}$, where, for any $F, G \in \Delta^{+}$and any real $x$,

$$
\tau_{T}(F, G)(x)=\sup _{u+v=x} T(F(u), G(v))
$$


and $T$ is a (left-continuous) t-norm, i.e., a (left-continuous) binary operation on the unit interval $[0,1]$ such that

(a) $T(a, 1)=a$,

(b) $T(c, d) \geqq T(a, b)$, whenever $c \geqq a, d \geqq b$,

(c) $T(a, b)=T(b, a)$,

(d) $T(T(a, b), c)=T(a, T(b, c))$.

In this paper we generally assume that $T$ is continuous. This implies that $\tau_{T}$ is continuous on $\Delta^{+}[17]$. The most important continuous $t$-norms are $\operatorname{Min}(a, b), \operatorname{Prod}(a, b)=a b$, and $T_{m}(a, b)=\operatorname{Max}\{a+b-1,0\}$.

Definition 1.1. A $t$-norm $T$ is Archimedean if it is continuous on $[0,1] \times[0,1]$ and such that $T(a, a)<a$ for all $a \in(0,1)$; and $T$ is strict if it is continuous on $[0,1] \times[0,1]$ and strictly increasing in each place on $(0,1] \times(0,1]$.

It is immediate that every strict $t$-norm is Archimedean. The $t$-norms of Definition 1.1 are completely characterized by the following representation theorem [7]:

THEOREM 1.1. The t-norm $T$ is Archimedean if and only if there exists a continuous and increasing function $h:[0,1] \rightarrow[0,1]$, with $h(1)=1$, such that

$$
T(a, b)=h^{[-1]}(h(a) \cdot h(b)),
$$

where

$$
h^{[-1]}(x)= \begin{cases}0, & 0 \leqq x \leqq h(0), \\ h^{-1}(x), & h(0) \leqq x \leqq 1\end{cases}
$$

and $h^{-1}$ is the usual inverse of $h$ on $[h(0), 1]$. Furthermore, $T$ is strict if and only if $h(0)=0$, in which case $h^{[-1]}=h^{-1}$.

The function $h$ in (1.5) is called a multiplicative generator of the $t$-norm $T$ and $h^{[-1]}$ is the pseudo-inverse of $h$.

2. Wald-betweenness. If $(S, d)$ is a metric space and $p, q, r$ are three distinct points of $S$ then $q$ is said to lie between $p$ and $r$-and one writes $p q r$-if and only if $d(p, r)=d(p, q)+d(q, r)$. This relation has the following properties [3]:

(B1) If $p q r$ then $r q p$.

(B2) If $p q r$ then neither $q r p$ nor $r p q$.

(B3) (a) If $p q r$ and $p r s$ then $p q s$.

(b) If $p q r$ and prs then $q r s$.

(B4) The set $B(p, r) \cup\{p, r\}$ is closed in the metric topology, where $B(p, r)=\{q \mid p q r\}$. 
In his paper [25] Wald considered the straightforward generalization of metric betweenness given by: If $p, q, r$ are three distinct points of a Wald space, then $q$ lies between $p$ and $r$ if and only if

$$
F_{p r}=F_{p q} * F_{q r},
$$

where * denotes convolution. In this section we consider Wald's betweenness for an arbitrary triangle function $\tau$.

Definition 2.1. Let $(S, \mathscr{F}, \tau)$ be a PM space and let $p, q, r$ be three distinct points of $S$. Then $q$ is Wald-between $p$ and $r$-and we write $W(p q r)$-if $F_{p r} \neq \varepsilon_{\infty}$ and

$$
F_{p r}=\tau\left(F_{p q}, F_{q r}\right) \text {, }
$$

where $\varepsilon_{\infty} \in \Delta^{+}$is defined by $\varepsilon_{\infty}(x)=0$ for all $x$.

Since $\varepsilon_{\infty}$ and $\varepsilon_{0}$ are, respectively, the mimimal and maximal elements of $\Delta^{+}$, for any $F \in \Delta^{+}$we have

$$
\varepsilon_{\infty} \leqq \tau\left(\varepsilon_{\infty}, F\right) \leqq \tau\left(\varepsilon_{\infty}, \varepsilon_{0}\right)=\varepsilon_{\infty}, .
$$

whence $\tau\left(\varepsilon_{\infty}, F\right)=\varepsilon_{\infty}$. Thus if $W(p q r)$ then none of $F_{p r}, F_{p q}, F_{q r}$ is equal to $\varepsilon_{\infty}$. Note also that, since $p, q, r$ are distinct, $W(p q r)$ implies that none of $F_{p r}, F_{p q}, F_{q r}$ is equal to $\varepsilon_{0}$.

LEMMA 2.1. Let the triangle function $\tau$ be continuous on $\Delta^{+}$. Then the following are equivalent:

(i) There exist $F, G \in \Delta^{+}$, both different from $\varepsilon_{\infty}$ and $\varepsilon_{0}$, such that $\tau(F, G)=F$.

(ii) There exists an $H \in \Delta^{+}$, different from $\varepsilon_{\infty}$ and $\varepsilon_{0}$, such that $\tau(H, H)=H$, i.e., there exists a nontrivial idempotent in the semi$\operatorname{group}\left(\Delta^{+}, \tau\right)$.

Proof. Clearly (ii) implies (i) on letting $F=G=H$. Therefore suppose (i) holds and let $G^{2}=\tau(G, G)$ and $G^{n+1}=\tau\left(G, G^{n}\right)$, for $n=$ $2,3, \cdots$. Then $\varepsilon_{0}>G \geqq G^{2} \geqq \cdots \geqq G^{n} \geqq \cdots$, whence the weak limit of the sequence $\left\{G^{n}\right\}$ exists and is distinct from $\varepsilon_{0}$. Denote this limit by $H$. Since $\tau$ is continuous, we have

$$
H=\lim _{n \rightarrow \infty} G^{2 n}=\lim _{n \rightarrow \infty} \tau\left(G^{n}, G^{n}\right)=\tau(H, H),
$$

whence $H$ is idempotent. Next, using (i) and the continuity of $\tau$ yields

$$
\begin{aligned}
F & =\tau(F, G)=\tau(\tau(F, G), G)=\tau\left(F, G^{2}\right) \\
& =\cdots=\tau\left(F, G^{n}\right)=\tau\left(F, \lim _{n \rightarrow \infty} G^{n}\right)=\tau(F, H) .
\end{aligned}
$$

Since $F \neq \varepsilon_{\infty}$, it follows from (2.3) that $H \neq \varepsilon_{\infty}$. Thus $H$ satisfies (ii) 
and the proof is complete.

CoROLlaRY 2.1. If $\tau$ is continuous then the semigroup $\left(\Delta^{+}, \tau\right)$ has no nontrivial idempotents if and only if

$$
\tau(F, G)<F
$$

whenever $F$ and $G$ are both different from $\varepsilon_{\infty}$ and $\varepsilon_{0}$.

On $\Delta^{+}$, the conditions (i) and (ii) of Lemma 2.1 are not satisfied by convolution nor by any $\tau_{T}$ when $T$ is Archimedean [11]. Thus these semigroups have no nontrivial idempotents.

Definition 2.2. A triangle function $\tau$ is strictly increasing on a subset $\mathscr{S}$ of $\Delta^{+}$if, for any $F, G, H \in \mathscr{S}, \tau(F, G)>\tau(F, H)$ whenever $F \neq \varepsilon_{\infty}$ and $G>H$.

It is easily seen that if the cancellation law holds in $\left(\Delta^{+}, \tau\right)$ then $\tau$ is strictly increasing on $\Delta^{+}$; and that if $\tau$ is strictly increasing on $\Delta^{+}$then $\left(\Delta^{+}, \tau\right)$ has no nontrival idempotents.

Theorem 2.1. Let $(S, \mathscr{F}, \tau)$ be a PM space. Then the Waldbetweenness relation:

(i) Always satisfies the betweenness properties (B1) and (B3a).

(ii) Satisfies (B2) whenever $\tau$ is continuous and has no nontrivial idempotents.

(iii) Satisfies (B2) and (B3b) whenever $\tau$ is strictly increasing on $\operatorname{Ran} \mathscr{F}$, the range of $\mathscr{F}$.

(iv) Satisfies (B4), with respect to the $\varepsilon$, $\lambda$-topology on $S$, whenever $\tau$ is continuous.

Proof. (i) The property (B1) is trivial. Now suppose $W(p q r)$ and $W(p r s)$. Then $F_{p r}=\tau\left(F_{p q}, F_{q r}\right), F_{p s}=\tau\left(F_{p r}, F_{r s}\right)$ and, in view of the triangle inequality, $F_{q s} \geqq \tau\left(F_{q r}, F_{r s}\right)$ and $F_{p s} \geqq \tau\left(F_{p q}, F_{q_{s}}\right)$. Thus

$$
\begin{aligned}
F_{p s}=\tau\left(\tau\left(F_{p q}, F_{q r}\right), F_{r s}\right) & =\tau\left(F_{p q}, \tau\left(F_{q r}, F_{r s}\right)\right) \\
& \leqq \tau\left(F_{p q}, F_{q s}\right) \leqq F_{p s}
\end{aligned}
$$

whence $W(p q s)$.

(ii) Suppose $W(p q r)$. Then, by the remarks after Definition 2.1, none of $F_{p q}, F_{q r}, F_{p r}$ is equal to either $\varepsilon_{0}$ or $\varepsilon_{\infty}$. Thus, using Corollary 2.1,

$$
\begin{aligned}
\tau\left(F_{q r}, F_{r p}\right) & =\tau\left(F_{q r}, \tau\left(F_{p q}, F_{q r}\right)\right) \\
& =\tau\left(F_{p q}, \tau\left(F_{q r}, F_{q r}\right)\right) \leqq \tau\left(F_{p q}, \tau\left(F_{q r}, \varepsilon_{0}\right)\right) \\
& =\tau\left(F_{p q}, F_{q r}\right)<F_{p q},
\end{aligned}
$$


whence $W(q r p)$ does not hold. Similarly $W(r p q)$ also does not hold.

(iii) To prove (B2), we have only to note that the display (2.6) remains valid.

Now suppose $W(p q r)$ and $W(p r s)$. If $F_{q s}>\tau\left(F_{q r}, F_{r s}\right)$ then the first inequality in (2.5) would be strict, which cannot be. Thus also $W(q r s)$, whence (B3b) holds.

(iv) Suppose that $\left\{q_{n}\right\}$ is a sequence in $B(p, r) \cup\{p, r\}$ such that $q_{n} \rightarrow q_{0} \in S$ in the $\varepsilon$, $\lambda$-topology. If $q_{0}=p$ or $q_{0}=r$, we are done. Otherwise the points $p, q_{0}, r$ are distinct and we may also assume without loss of generality that all the triples $\left(p, q_{n}, r\right)$ consist of distinct points, so that $W\left(p q_{n} r\right)$ for all $n$. Then, using the fact that $F_{q_{0} \eta_{n}} \stackrel{w}{\rightarrow} \varepsilon_{0}$, we have

$$
\begin{aligned}
F_{p_{r}} & \geqq \tau\left(F_{p q_{0}}, F_{q_{0} r}\right) \geqq \tau\left(\tau\left(F_{p q_{n}}, F_{q_{n} q_{0}}\right), \tau\left(F_{q_{0} q_{n}}, F_{q_{n} r}\right)\right) \\
& =\tau\left(\tau\left(F_{p q_{n}}, F_{q_{n} r}\right), \tau\left(F_{q_{0} q_{n}}, F_{q_{0} q_{n}}\right)\right) \\
& =\tau\left(F_{p r}, \tau\left(F_{q_{0} q_{n}}, F_{q_{0} q_{n}}\right)\right) \stackrel{w}{\longrightarrow} F_{p_{r}},
\end{aligned}
$$

since $\tau$ is continuous. Thus $W\left(p q_{0} r\right)$, whence $q_{0} \in B(p, r)$, and the proof is complete.

Note. The proofs of (i) and (iii) are generalizations of Wald's arguments [25], and the proof of (iv) is a generalization of the argument used by F. Rhodes in [16].

Theorem 2.1 applies in the following special cases:

(i) $\tau=$ convolution. In this case Wald [25] has shown that $\tau$ is strictly increasing on the subset $\mathscr{D}^{+}$of $\Delta^{+}$given by

$$
\mathscr{D}^{+}=\left\{F \in \Delta^{+} \mid \lim _{x \rightarrow \infty} F(x)=1\right\},
$$

and Wald's argument extends to $\Delta^{+}$. Equivalently, this follows from the validity of the cancellation law in the semigroup $\left(\Delta^{+}, *\right)$.

(ii) $\tau=\tau_{M \text { in }}$ and $\operatorname{Ran} \mathscr{F}^{-} \subset \mathscr{D}^{+}$. In this case, as was shown in [9], the cancellation law holds in the semigroup $\left(\mathscr{D}^{+}, \tau_{\mathrm{Min}}\right)$.

For example, if $(\mathscr{P}, \mathscr{F})$ is the simple space generated by the metric space $(S, d)$ and the distribution function $G \in \mathscr{D}^{+}$, so that for any distinct $p, q \in S$,

$$
F_{p q}(x)=G(x / d(p, q)),
$$

then $(S, \mathscr{F})$ is a Menger space under $\tau_{\mathrm{Min}}[18]$. Moreover, it is easy to show that in this case Wald-betweenness and $d$-metric betweenness are equivalent.

(iii) $\tau=\tau_{T}$ where $T$ is a strict $t$-norm and $\operatorname{Ran} \mathscr{T}_{\subseteq} \leqq \Delta_{T}^{+}$(see Definition 3.2). In this case the cancellation law holds in $\left(\Delta_{T}^{+}, \tau_{T}\right)$ [10]. 
The following examples show that the relation $W(p q r)$ may fail to satisfy either (B2) or (B3b) when the corresponding hypotheses of Theorem 2.1 are not satisfied.

EXAmPLe 2.1. Let $\tau$ be a triangle function for which there exists a nontrivial $H \in \Delta^{+}$such that $\tau(H, H)=H$. Let $(S, \mathscr{F})$ be the equilateral PM space in which $F_{p q}=H$ for any pair of distinct points $p$ and $q$. Then $W(p q r)$ holds for all triples of distinct points in $S$ and thus (B2) fails.

EXAMPLE 2.2. In [9] it was shown that the cancellation law fails in the semigroup $\left(\Delta^{+}, \tau_{\text {Prod }}\right)$. The counterexample which established this fact will serve us here as well. Let $S=\{p, q, r, s\}$ and first define $\mathscr{F}$ via:

$$
\begin{aligned}
& F_{p q}(x)= \begin{cases}0, & x \leqq 0, \\
x, & 0 \leqq x \leqq 1, \\
1, & 1 \leqq x ;\end{cases} \\
& F_{q r}(x)=\varepsilon_{0}(x-1) ; \\
& F_{r s}(x)= \begin{cases}0, & x \leqq 0, \\
1 / 2, & 0<x \leqq 1.45, \\
1, & 1.45<x ;\end{cases}
\end{aligned}
$$

and $F_{p r}=\tau_{\text {Prod }}\left(F_{p q}, F_{q r}\right), F_{p s}=\tau_{\text {Prod }}\left(F_{p r}, F_{r s}\right), F_{q_{s}}=\tau_{\text {Prod }}\left(F_{q r}, F_{r s}\right)$. Now, by (i) of Theorem 2.1, it follows that

$$
F_{p s}=\tau_{\text {Prod }}\left(F_{p q}, F_{q s}\right),
$$

whence we have $W(p q r), W(p r s), W(q r s)$, and $W(p q s)$. Using the above, it is easily verified that $\left(S, \mathscr{F}, \tau_{\text {Prod }}\right)$ is a PM space in which Wald-betweenness satisfies (B1)-(B4). However, as shown in [9], we can alter $F_{q s}$ slightly and still maintain equality in (2.9). In fact, if $G \in \Delta^{+}$is given by

$$
G(x)= \begin{cases}0, & x \leqq 1 \\ 1 / 2, & 1<x \leqq 2.4 \\ .55, & 2.4<x \leqq 2.45 \\ 1, & 2.45<x\end{cases}
$$

then $G>F_{q s}$, but $\tau_{\text {Prod }}\left(F_{p q}, G\right)=\tau_{\text {Prod }}\left(F_{p q}, F_{q s}\right)$. Thus, if we let $F_{q s}^{\prime}=G$ and let the remaining distance distribution functions be defined as before then we obtain a space $\left(S, \mathscr{F}^{\prime}\right)$ which is still a PM space under $\tau_{\text {Prod }}$. Furthermore in this new space $W(p q r), W(p r s)$, and $W(p q s)$ hold but, by construction, $W(q r s)$, and hence (B3b), fails. 
Using some of the results of [9], similar counterexamples can be constructed for any triangle function $\tau_{T}$, where $T$ is a continuous $t$-norm.

We conclude this section with an interesting particular instance of Wald betweenness.

Let $(S, \mathscr{F})$ be a pseudo-metrically generated PM space, that is to say, let $S$ be a given set, let $(D, \mathscr{B}, \mu)$ be a probability space whose elements are pseudo-metrics on $S$, and let $\mathscr{F}$ be defined via

$$
F_{p q}(x)=\mu\{d \in D \mid d(p, q)<x\} .
$$

Then $(S, \mathscr{F})$ is a Menger space under $\tau_{T_{m}}$ [22]. Furthermore, $T_{m}$ is the strongest $t$-norm for the class of pseudo-metrically generated spaces [4]. (Note: This does not mean that $T_{m}$ is the strongest $t$ norm for all such spaces. For example, simple spaces, which are Menger spaces under Min, are pseudo-metrically generated. In general, for Wald-betweenness to be a meaningful relation in a specific Menger space, it must be with respect to the strongest $t$-norm for that space.)

As regards Wald-betweenness with respect to $\tau_{T_{m}}$, we have the following:

THEOREM 2.2. If $(S, \mathscr{F})$ is a pseudo-metrically generated $P M$ space and $p, q, r$ are distinct points of $S$, then $q$ is Wald-between $p$ and $r$, i.e., $W(p q r)$ holds with respect to $\tau_{T_{m}}$, if and only if $q$ is between $p$ and $r$ for almost all pseudo-metrics $d$ in the generating collection $D$ and either $d(p, q)$ or $d(q, r)$ is constant for almost all $d$ in $D$.

Proof. For any $p, q \in S$, the mapping $(p q): D \rightarrow \boldsymbol{R}^{+}$defined by $(p q)(d)=d(p, q)$ is a nonnegative random variable whose distribution function is the function $F_{p q}$ given by (2.10). Note that $F_{p q} \in \mathscr{D}^{+}$, where $\mathscr{D}^{+}$is given by (2.7). Furthermore, for any $p, q, r \in S$ the joint distribution function of $(p q)$ and $(q r)$ exists and is given by

$$
F_{p q, q r}(u, v)=\mu\{d \mid d(p, q)<u, d(q, r)<v\} .
$$

Let $C$ be the connecting copula of $(p q)$ and $(q r)$ [20], so that

$$
F_{p q, q r}(u, v)=C\left(F_{p q}(u), F_{q r}(v)\right) \text {. }
$$

Then the distribution function of the random variable $(p q)+(q r)$, i.e., $d(p, q)+d(q, r)$, is given by

$$
F_{p q+q r}=\sigma_{C}\left(F_{p q}, F_{q r}\right),
$$

where $\sigma_{C}$ is the binary operation on $\Delta^{+}$defined via

$$
\sigma_{C}\left(F_{p q}, F_{q r}\right)(x)=\iint_{u+v<x} d C\left(F_{p q}(u), F_{q r}(v)\right) \text {. }
$$


Since each of the pseudo-metrics $d \in D$ satisfies the ordinary triangle inequality, we have

$$
\begin{aligned}
F_{p q+q r}(t) & =\mu\{d \mid d(p, q)+d(q, r)<t\} \\
& \leqq \mu\{d \mid d(p, r)<t\}=F_{p r}(t) .
\end{aligned}
$$

So much holds in general. To proceed with the proof, suppose first that $W(p q r)$, i.e., that $F_{p r}=\tau_{T_{m}}\left(F_{p q}, F_{q r}\right)$. Then (2.11) and (2.12) yield

$$
\sigma_{C}\left(F_{p q}, F_{q r}\right) \leqq \tau_{T_{m}}\left(F_{p q}, F_{q r}\right) \text {. }
$$

Now it is known that $\tau_{T_{m}}(F, G) \leqq \sigma_{C}(F, G)$ for any $F, G \in \Delta^{+}[17]$. Hence

$$
\sigma_{C}\left(F_{p q}, F_{q r}\right)=\tau_{T_{m}}\left(F_{p q}, F_{q r}\right) .
$$

But $F_{p q}, F_{q r}$ belong to the subspace $\mathscr{D}^{+}$of $\Delta^{+}$; and in this case it can be shown ${ }^{1}$ that (2.13) holds if and only if, for some $a, b>0$, either $F_{p q}=\varepsilon_{a}$ or $F_{q r}=\varepsilon_{b}$, where $\varepsilon_{a}(x)=\varepsilon_{0}(x-a), \varepsilon_{b}(x)=\varepsilon_{0}(x-b)$. Thus for almost all $d \in D$, either $d(p, q)=a$ or $d(q, r)=b$. Suppose $d(p, q)=a$. Then

$$
F_{p r}(x)=\tau_{T_{m}}\left(\varepsilon_{a}, F_{q r}\right)(x)=F_{q r}(x-a),
$$

which, combined with the fact that $d(p, r) \leqq d(p, q)+d(q, r)$, yields $d(p, r)=a+d(q, r)$ for almost all $d \in D$. Similarly, if $d(q, r)=b$ then $d(p, r)=d(p, q)+b$ for almost all $d \in D$. This proves the first half of the theorem.

In the other direction, suppose that $d(p, r)=d(p, q)+d(q, r)$ and $d(p, q)=a>0$, for almost all $d \in D$. Then $F_{p q}=\varepsilon_{a}$ and

$$
F_{p r}(x)=F_{q r}(x-a)=\tau_{T_{m}}\left(F_{p q}, F_{q r}\right)(x),
$$

whence $W(p q r)$; and similarly if $d(q, r)=b>0$.

COROLlARY 2.2. In a pseudo-metrically generated PM space Wald-betweenness, with respect to $\tau_{T_{m}}$, satisfies all the properties of metric betweenness.

Proof. Since $\tau_{T_{m}}$ is continuous and has no nontrivial idempotents, only (B3b) needs verification. But this is immediate.

To illustrate Theorem 2.2, let $L$ be the set of all Lebesgue measurable functions on $[0,1]$. For any $t \in[0,1]$ and any $f, g \in L$, let $d_{t}$ be the pseudo-metric on $L$ defined by

$$
d_{t}(f, g)=|f(t)-g(t)| \text {. }
$$

\footnotetext{
1 The proof, which is rather lengthy, is given in [12; Theorem 7 and Corollary].
} 
Let $D=\left\{d_{t} \mid t \in[0,1]\right\}$, let $\mu$ be the measure on $D$ induced by Lebesgue measure $\lambda$ on $[0,1]$ and let $\mathscr{F}$ be defined via

$$
F_{f g}(x)=\mu\left\{d_{t} \mid d_{t}(f, g)<x\right\}=\lambda\{t|| f(t)-g(t) \mid<x\} .
$$

Then $(L, \mathscr{F})$ is a pseudo-metrically generated space-and it is easy to show that $T_{m}$ is the strongest $t$-norm under which it is a Menger space. Furthermore, for any distinct $f, g, h$ in $L, g$ is Wald-between $f$ and $h$ if and only if the graph of $g$ lies between the graph of $f$ and the graph of $h$ almost everywhere and either $|f(t)-g(t)|=a>0$ almost everywhere or $|g(t)-h(t)|=b>0$ almost everywhere. In particular, if $f(t)=h(t)$ on a set of positive measure then there is no $g$ such that $W(f g h)$. It follows that in $L$ Wald-betweenness is considerably stronger than betweenness with respect to either the $L_{\infty}$ (ess sup) metric or the $L_{1}$ metric ( $L_{1}$ betweenness is simply pointwise almost-everywhere betweenness). However, for $1<p<\infty$, Wald-betweenness is not comparable to betweenness with respect to the $L_{p}$ metric.

The space in the above example is an $E$-space [22]; and since any $E$-space is a pseudo-metrically generated space, the above discussion generalizes at once to yield:

CoRollary 2.3. Let $(S, \mathscr{F})$ be an E-space, of mappings from the probability space $(\Omega, \mathscr{A}, P)$ into the metric space $(M, d)$. Let $p, q, r$ be distinct elements of $S$. Then $W(p q r)$ if and only if $q(t)$ is between $p(t)$ and $r(t)$ in $(M, d)$ for almost all $t \in \Omega$ and either. $d(p(t), q(t))=a>0$ for almost all $t \in \Omega$ or $d(q(t), r(t))=b>0$ for almost all $t \in \Omega$.

3. Envelope-betweenness. It is desirable to have a betweenness relation which satisfies (B1)-(B4) even when the triangle function $\tau$ is not strictly increasing. When $\tau$ is of the form $\tau_{T}$, for some Archimedean $t$-norm $T$, such a relation exists. In order to define and study it, we need some of the elements of the theory of the conjugate transform for $\tau_{T}$-semigroups. ${ }^{2}$ This is the analog of the Laplace transform for the convolution semigroup $\left(\Delta^{+}, *\right)$.

Throughout the rest of this paper, unless explicitly state otherwise, $T$ will denote an Archimedean $t$-norm, $h$ a fixed multiplicative generator of $T$, and $h^{[-1]}$ the pseudo-inverse of $h$.

${ }^{2}$ The conjugate transform was first defined by W. Fenchel [5] and later, independently, by R. Bellman and W. Karush [1, 2] who also developed many of its properties. Their results apply directly to the semigroup $\left(\Delta^{+}, \tau_{\text {Prod }}\right)$. The development of the theory of this transform, its inverse transform, limit theorems, etc., for the semigroups $\left(\Delta^{+}, \tau_{T}\right)$, when $T$ is an arbitrary Archimedean $t$-norm, is the central topic of [10]. The details are given in [13], [14], and [15]. 
DEFINITION 3.1. The T-conjugate transform for the semigroup $\left(\Delta^{+}, \tau_{T}\right)$ is the mapping $C_{T}$ defined for any $F \in \Delta^{+}$via:

$$
C_{T} F(z)=\sup _{x \geqq 0} e^{-x z} h F(x), \quad \text { for all } z \geqq 0,
$$

where $h F \in \Delta^{+}$is given by

$$
h F(x)=\left\{\begin{array}{cl}
0, & x \leqq 0 \\
h(F(x)), & 0<x
\end{array}\right.
$$

T-conjugate transforms are completely characterized by the following:

THEOREM 3.1. Let $A_{T}=\left\{C_{T} F \mid F \in \Delta^{+}\right\}$. Then (3.3) $\mathscr{A}_{T}=\{\phi:[0, \infty) \longrightarrow[h(0), 1] \mid \phi$ is nonincreasing, positive, continuous and log-convex $\} \cup\left\{\theta_{T}\right\}$,

where $\theta_{T}(z)=h(0)$ for all $z \geqq 0$.

Definition 3.2. (i) $C_{T}^{*}$ is the mapping defined for any $\phi \in \mathscr{A}_{T}$ via

$$
C_{T}^{*} \phi(x)=h^{[-1]}\left(\inf _{z \geqq 0} e^{x z} \phi(z)\right), \quad \text { for all } x,
$$

and where, in addition, $C_{T}^{*} \phi$ is normalized so as to be left-continuous.

(ii) $F \in \Delta^{+}$is $T$-log-concave if $\log (h F)$ is concave on $\left(b_{F}, \infty\right)$, where

$$
b_{F}=\sup \{x \mid h F(x)=0\} .
$$

Furthermore,

$$
\Delta_{*}=\left\{F \in \Delta^{+} \mid F \text { is } T \text {-log-concave }\right\} \text {. }
$$

(iii) For any $F \in \Delta^{+}, \overline{h F}$ is the function with the following properties: $\overline{h F}(x)=0$ for $x \leqq b_{F}$; on $\left(b_{T}, \infty\right)$ the graph of $\log (\overline{h F})$ is the concave hull of the graph of $\log (h F)$.

(iv) For any $F \in \Delta^{+}$, the $T$-log-concave envelope of $F$ is the function $F_{T}$ in $\Delta_{T}^{+}$given by

$$
F_{T}=h^{[-1]}(\overline{h F}) .
$$

REMARK. The conjugate transform $C_{T}$ defined by (3.1) clearly depends on the choice of multiplicative generator $h$. However, any other multiplicative generator of $T$ is of the form $h^{\lambda}$, for some $\lambda>0$. From this it follows that $T$-log-concavity is independent of the par- 
ticular choice of multiplicative generator, whence $\Delta_{T^{\prime}}^{+}$is completely determined by $T$ alone. Similarly, $T$-log-concave envelopes depend only on $T$. Furthermore, if $C_{T}^{\prime}$ is the conjugate transform determined by $h^{\lambda}$, for some $\lambda>0$, then for any $F \in \Delta^{+}$,

$$
C_{T}^{\prime} F(z)=\left[C_{T} F(z / \lambda)\right]^{\lambda},
$$

whence $\log C_{T}^{\prime} F(z)=\lambda \log C_{T} F(z / \lambda)$ so that the transforms determined by distinct multiplicative generators of the same $t$-norm are essentially equivalent.

The elements of the theory of the $T$-conjugate transform which will be needed in the sequel are listed in:

Theorem 3.2. For any $F, G, H \in \Delta^{+}$and any $\phi, \theta \in A_{T}$, we have: (C 1) $C_{T} \tau_{T}(F, G)(z)=\max \left[h(0), C_{T} F(z) \cdot C_{T} G(z)\right]$, for all $z \geqq 0$. Thus, if $T$ is strict, $C_{T} \tau_{T}(F, G)=C_{T} F \cdot C_{T} G$.

(C 2) $C_{T}: \Delta_{T}^{+} \rightarrow \mathscr{A}_{T}$ is one-one, onto, with inverse $C_{T}^{*}$.

(C 3) If $F \geqq G$ then $C_{T} F \geqq C_{T} G$.

(C 4) If $\phi \geqq \theta$ then $C_{T}^{*} \dot{\theta} \geqq C_{T}^{*} \theta$.

(C 5) $\quad F_{T} \geqq F$.

(C 6) If $F \geqq G$ then $F_{T} \geqq G_{T}$.

(C 7) $C_{T} F_{T}=C_{T} F$.

(C 8) $C_{T}^{*} C_{T} F=F_{T}$.

(C 9) If $F \in \Delta_{T}$ then $F_{T}=F$.

(C10) $\tau_{T}\left(F_{T}, G_{T}\right)$ is T-log-concave if and only if $C_{T}\left(\tau_{T}(F, G)\right)=$ $C_{T} F \cdot C_{T} G$.

(C11) $\quad C_{T}^{*}\left(C_{T} F \cdot C_{T} G\right)=\tau_{T}\left(F_{T}, G_{T}\right)$.

(C12) $\left(\tau_{T}(F, G)\right)_{T} \geqq \tau_{T}\left(F_{T}, G_{T}\right)$, with equality if $T$ is strict.

(C13) If $\tau_{T}\left(F_{T}, G_{T}\right)=\tau_{T}\left(F_{T}, H_{T}\right) \neq \varepsilon_{\infty}$ and is T-log-concave then $G_{T}=H_{T}$.

(C14) If $T$ is strict then $\left(\Delta_{T}^{+}, \tau_{T}\right)$ is a subsemigroup in which the cancellation law holds.

(C15) If, for some $a>0, G(x)=F(a x)$, for all $x$, then $C_{T} G(z)$ $=C_{T} F(z / a)$, for all $z \geqq 0$.

(C16) If $F_{n} \stackrel{w}{\rightarrow} F$ then $C_{T} F_{n}(z) \rightarrow C_{T} F(z)$, for all $z>0$.

(C17) For any $z>0, C_{T} F_{n}(z) \rightarrow 1$ if and only if $F_{n} \stackrel{w}{\rightarrow} \varepsilon_{0}$.

(C18) For any $z>0, C_{T} F(z)=1$ if and only if $F=\varepsilon_{0}$.

(C19) $F_{T}=\varepsilon_{0}$ if and only if $F=\varepsilon_{0}$.

(C20) $F_{T}=\varepsilon_{\infty}$ if and only if $F=\varepsilon_{\infty}$.

Perusal of the above shows that the essential properties of $T$ conjugate transforms, as well as their usefullness as analytical tools, are independent of the particular choice of multiplicative generator in (3.1). 
Definition 3.3. Let $\left(S, \mathscr{F}, \tau_{T}\right)$ be a PM space where $T$ is an Archimedean $t$-norm. Let $p, q, r$ be distinct points of $S$. Then $q$ is envelope-between $p$ and $r$-and we write $E(p q r)$-if $F_{p r} \neq \varepsilon_{\infty}$ and

$$
\left(F_{p r}\right)_{T}=\tau_{T}\left(\left(F_{p q}\right)_{T},\left(F_{q r}\right)_{T}\right),
$$

where, for any $F \in J^{+}, F_{T}$ is the $T$-log-concave-envelope of $F$.

Again, $E(p q r)$ implies that $F_{p r}, F_{p q}$, and $F_{q r}$ are all different from both $\varepsilon_{\infty}$ and $\varepsilon_{0}$.

THEOREM 3.3. Let $\left(S, \mathscr{F}, \tau_{T}\right)$ be a $P M$ space, where $T$ is an Archimedean t-norm. Then the betweenness relation $E(p q r)$ satisfies (B1)-(B4).

Proof. To simplify the notation, we will denote the $T$-log-concave envelope $F_{T}$ of any $F \in \Delta^{+}$by $\bar{F}$ and $\tau_{T}, C_{T}$ by $\tau$ and $C$, respectively.

Again (B1) is trivial. To prove (B2) we merely replace the distribution functions in (2.6) by their $T$-log-concave envelopes. As noted previously, Corollary 2.1 applies to $\left(\Delta^{+}, \tau_{T}\right)$ when $T$ is Archimedean and (C19), (C20) imply that none of $\bar{F}_{p q}, \bar{F}_{q r}, \bar{F}_{p r}$ is equal to either $\varepsilon_{0}$ or $\varepsilon_{\infty}$.

To establish (B3) suppose $E(p q r)$ and $E(p r s)$ hold, so that

$$
\bar{F}_{p r}=\tau\left(\bar{F}_{p q}, \bar{F}_{p r}\right) \text { and } \bar{F}_{p s}=\tau\left(\bar{F}_{p r}, \bar{F}_{r s}\right) .
$$

Note that, by (III), (C6) and (C12), we have

$$
\bar{F}_{q s} \geqq \overline{\tau\left(F_{q r}, F_{r s}\right)} \geqq \tau\left(\bar{F}_{q r}, \bar{F}_{r s}\right)
$$

and, similarly, $\bar{F}_{p s} \geqq \tau\left(\bar{F}_{p q}, \bar{F}_{q s}\right)$. Hence, replacing the distribution functions in (2.5) by their $T$-log-concave envelopes yields $E(p q s)$. Next, using this fact, (C7) and (C10), we have

$$
\begin{aligned}
C F_{p q} \cdot C F_{q s} & =C \tau\left(\bar{F}_{p q}, \bar{F}_{q s}\right)=C \bar{F}_{p s} \\
& =C \tau\left(\bar{F}_{p r}, \bar{F}_{r s}\right)=C F_{p r} \cdot C F_{r s} \\
& =C \tau\left(\bar{F}_{p q}, \bar{F}_{q r}\right) \cdot C F_{r s}=C F_{p q} \cdot C F_{q r} \cdot C F_{r s} .
\end{aligned}
$$

Since $F_{p q} \neq \varepsilon_{\infty}$, it follows from (3.1) that $C F_{p q}(z)>0$ for all $z \geqq 0$. Thus, cancelling $C F_{p q}$ in (3.10) yields:

$$
C F_{q s}=C F_{q r} \cdot C F_{r s},
$$

whence, by (C8) and (C11), we have

$$
\bar{F}_{q s}=C^{*} C F_{q s}=C^{*}\left(C F_{q r} \cdot C F_{r s}\right)=\tau\left(\bar{F}_{q r}, \bar{F}_{r s}\right)
$$

and $E(q r s)$. 
The proof of (B4) follows as in Theorem 2.1 (iv), using the facts that:

(i) $\tau_{T}$ is continuous with respect to weak convergence on $\Delta^{+}$;

(ii) taking $T$-log-concave envelopes is a continuous operation, i.e., if $F_{q_{0} q_{n}} \stackrel{w}{\rightarrow} \varepsilon_{0}$ then $\bar{F}_{q_{0} q_{n}} \stackrel{w}{\rightarrow} \bar{\varepsilon}_{0}=\varepsilon_{0}$; and

(iii) $\bar{F}_{p r} \geqq \tau\left(\bar{F}_{p q}, \bar{F}_{q r}\right)$ for any $p, q, r \in S$. This completes the proof.

CoRollary 3.1. Let $\mathscr{F}_{T}$ be the mapping defined on $S \times S$ by $\mathscr{F}_{T}(p, q)=(\mathscr{F}(p, q))_{T}$. Then $\left(S, \mathscr{F}_{T}, \tau_{T}\right)$ is also a PM space. Furthermore, in $\left(S, \mathscr{F}_{T}, \tau_{T}\right)$ Wald-betweenness and envelope-betweenness coincide.

Note that Wald-betweenness and envelope-betweenness coincide in any $\mathrm{PM}$ space $\left(S, \mathscr{F}, \tau_{T}\right)$ for which $\operatorname{Ran} \mathscr{F} \subseteq \Delta_{T}^{+}$.

For strict $t$-norms, Wald-betweenness is stronger than envelopebetweenness, for we have:

TheOREM 3.4. Let $\left(S, \mathscr{F}, \tau_{T}\right)$ be a PM space, where $T$ is a strict t-norm. Then, for any distinct $p, q, r$ in $S, W(p q r)$ implies $E(p q r)$.

Proof. Suppose $W(p q r)$ so that $F_{p r}=\tau_{T}\left(F_{p q}, F_{q r}\right)$. Then, using (C12), we have

$$
\left(F_{p r}\right)_{T}=\left(\tau_{T}\left(F_{p q}, F_{q r}\right)\right)_{T}=\tau_{T}\left(\left(F_{p q}\right)_{T},\left(F_{q r}\right)_{T}\right) .
$$

In PM spaces $\left(S, \mathscr{F}, \tau_{T}\right)$, where $T$ is Archimedean but not strict, the relations $W(p q r)$ and $E(p q r)$ are generally not comparable.

4. Conjugate-metric-betweenness. In [6] J. F. C. Kingman showed that in a Wald space the function $d$ defined on $S \times S$ by

$$
d(p, q)=-\log \left[\int_{0}^{\infty} e^{-x} d F_{p q}(x)\right]
$$

is a metric on $S$; that $d$-metric beteennesss and Wald-betweenness are equivalent; and that the $d$-metric topology and the $\varepsilon, \lambda$-topology are also equivalent.

The right-hand side of (4.1) is just the negative of the logarithm of the Laplace transform of $F_{p q}$ evaluated at 1 ; and since the $T$ conjugate transform is related to the semigroup $\left(\Delta^{+}, \tau_{T}\right)$ just as the Laplace transform is related to $\left(\Delta^{+}, *\right)$, we are led to the following:

THEOREM 4.1. Let $\left(S, \mathscr{Y}, \tau_{T}\right)$ be a PM space, with T Archimedean, and such that $F_{p q} \neq \varepsilon_{\infty}$ for any $p, q \in S$. Let $C_{T}$ be the T-conjugate transform on $\left(\Delta^{+}, \tau_{T}\right)$, as given by (3.1); and for any $z>0$ let $d_{z}$ be 
the mapping defined on $S \times S$ by

$$
d_{z}(p, q)=-\log C_{T} F_{p q}(z) .
$$

Then $d_{z}$ is a metric on $S$. Furthermore, the $d_{z}$-metric topology is equivalent to the $\varepsilon, \lambda$-topology.

Proof. ${ }^{3} \quad$ By (3.3), clearly $0 \leqq d_{z}(p, q)<\infty$ for any $p, q \in S$. If $d_{z}(p, q)=0$ then $C F_{p q}(z)=1$, whence by (C18) $F_{p q}=\varepsilon_{0}$ and $p=q$. Conversely, if $p=q$ then $d_{z}(p, q)=0$.

Since $F_{p q}=F_{q p}$ we have $d_{z}(p, q)=d_{z}(q, p)$.

For any $p, q, r \in S$, using (III), (C3), and (C1), we have

$$
C F_{p r}(z) \geqq C\left(\tau\left(F_{p q}, F_{q r}\right)\right)(z) \geqq C F_{p q}(z) \cdot C F_{q r}(z),
$$

whence, using (4.2), $d_{z}(p, r) \leqq d_{z}(p, q)+d_{z}(q, r)$. Thus $d_{z}$ is a metric on $S$.

Next let $\left\{p_{n}\right\}$ be a sequence in $S$ and let $p \in S$. Then, by (4.2) and (C17), the following are equivalent:

(i) $d_{z}\left(p_{n}, p\right) \rightarrow 0$;

(ii) $C F_{p_{n} p}(z) \rightarrow 1$;

(iii) $F_{p_{n} p} \stackrel{w}{\rightarrow} \varepsilon_{0}$;

(iv) $p_{n} \rightarrow p$ in the $\varepsilon, \lambda$-topology.

This completes the proof.

For any $z>0$ the function $d_{z}$ defined by (4.2) will be called a conjugate-metric on $S$. Clearly, any two conjugate metrics induce the same topology on $S$. But more is true:

THEOREM 4.2. Under the hypotheses of Theorem 4.1, if $z \geqq w$ $>0$ then, for any $p, q \in S$,

$$
d_{w}(p, q) \leqq d_{z}(p, q) \leqq \frac{z}{w} d_{w}(p, q) .
$$

Proof. The first inequality in (4.3) follows immediately from the fact that $C F_{p q}$ is nonincreasing. Next, since the function $f(y)=$ $-\log C F_{p q}(y)$ is concave and nonnegative on $[0, \infty)$, we have

$$
(f(w)-f(0)) / w \geqq(f(z)-f(0)) / z .
$$

Thus $z f(w) \geqq w f(z)+(z-w) f(0) \geqq w f(z)$, which yields the second inequality in (4.3) and completes the proof.

It follows from (4.3) that for any $z, w>0$, the conjugate metrics $d_{z}, d_{w}$ are equivalent.

${ }^{3}$ As in the proof of Theorem 3.3, in the proofs given in this section we suppress reference to the subscript $T$, and denote $F_{T}$ by $\vec{F}$. 
Definition 4.1. Suppose the hypotheses of Theorem 4.1 are satisfied and let $p, q, r$ be three distinct points of $S$. Then $q$ is between $p$ and $r$ with respect to the conjugate metric $d_{z}$-and we write $M_{z}(p q r)$-if $d_{z}(p, r)=d_{z}(p, q)+d_{z}(q, r)$.

Since $M_{z}(p q r)$ is a metric betweenness relation, it is immediate that (B1)-(B4) are satisfied. There is also a connection between the relations $\left\{M_{z}(p q r) \mid z>0\right\}$ and envelope-betweenness $E(p q r)$.

THEOREM 4.3. Let $\left(S, \mathscr{F}, \tau_{T}\right)$ be a PM space, with T Archimedean. Then, for any distinct $p, q, r \in S, E(p q r)$ if and only if $M_{z}(p q r)$ for all $z>0$.

Proof. Suppose $E(p q r)$, so that $\bar{F}_{p r}=\tau\left(\bar{F}_{p q}, \bar{F}_{q r}\right)$. Then, by (C7) and (C10), we have

$$
\begin{aligned}
C F_{p r}=C \bar{F}_{p r} & =C \tau\left(\bar{F}_{p q}, \bar{F}_{q r}\right) \\
& =C \bar{F}_{p q} \cdot C \bar{F}_{q r}=C F_{p q} \cdot C F_{q r},
\end{aligned}
$$

whence it follows from (4.2) and Definition 4.1 that $M_{z}(p q r)$ for all $z>0$.

Conversely, if $M_{z}(p q r)$ holds for all $z>0$, then for all $z>0$ we have

$$
C F_{p r}(z)=C F_{p q}(z) \cdot C F_{q r}(z),
$$

and the continuity of the conjugate transform on $[0, \infty)$ yields (4.4) at $z=0$, and hence for all $z \geqq 0$. Thus, using (C8) and (C11), we have

$$
\bar{F}_{p r}=C^{*} C F_{p r}=C^{*}\left(C F_{p q} \cdot C F_{q r}\right)=\tau\left(\bar{F}_{p q}, \bar{F}_{q r}\right),
$$

whence $E(p q r)$.

COROLlaRY 4.1. If $T$ is strict then, for any distinct $p, q, r \in S$, $W(p q r)$ implies $M_{z}(p q r)$ for all $z>0$.

In the case of convolution the betweenness relations obtained via (4.1) are independent of the particular point at which the Laplace transform is evaluated. In contrast, there are virtually no connections among the conjugate metric betweenness relations $\left\{M_{z}(p q r)\right\}$ for different values of $z$. This is brought out by the following example:

EXAMPLE 4.1. Let $S=\{p, q, r\}$ and let $\tau=\tau_{\text {Prod. }}$. Let $\phi(z)=$ $\exp (-\sqrt{z})$, for $z \geqq 0$. Then $\phi$ is a Prod-conjugate transform. Let 
$F_{p q}=F_{q r}=C^{*} \phi$. To define $F_{p r}$ we proceed as follows: Choose a fixed $w>0$ and let

$$
f(z)= \begin{cases}(-2 / \sqrt{w}) z, & 0 \leqq z \leqq w \\ -2 \sqrt{w}, & w \leqq z\end{cases}
$$

Thus $f$ is linear between the points $(0,0)$ and $(w,-2 \sqrt{w})$ and constant thereafter. Note also that the points $(0,0)$ and $(w,-2 \sqrt{w})$ lie on the graph of

$$
-2 \sqrt{z}=\log \left(\phi^{2}(z)\right)=\log \left(C F_{p q}(z) \cdot C F_{q r}(z)\right) .
$$

In particular, $f(z) \geqq-2 \sqrt{z}$ and, since $f$ is convex on $[0, \infty)$, the function $\theta(z)=\exp (f(z))$ is also a Prod-conjugate transform. Let $F_{p r}=C^{*} \theta$. Then we have

$$
C F_{p r}(z)=\theta(z) \geqq C F_{p q}(z) \cdot C F_{q r}(z), \quad \text { for all } z \geqq 0 .
$$

Using (C11), it follows that

$$
F_{p r}=C^{*} \theta \geqq C^{*}\left(C F_{p q} \cdot C F_{q r}\right)=\tau\left(F_{p q}, F_{q r}\right),
$$

which, together with the obvious inequalities $F_{p q}=\tau\left(\varepsilon_{0}, F_{q r}\right) \geqq$ $\tau\left(F_{p r}, F_{r q}\right)$ and $F_{q r} \geqq \tau\left(F_{q p}, F_{p r}\right)$, yields that $S$ is a PM space under $\tau_{\text {Prod. }}$. However, by construction, equality in (4.5) holds only when $z=0$ or $z=w$. Thus $M_{w}(p q r)$ holds while $M_{z}(p q r)$ fails for any other $z>0$.

The above example can obviously be modified so that $M_{z}(p q r)$ holds for any $z$ in the finite set of positive numbers $\left\{w_{1}, \cdots, w_{n}\right\}$ and fails otherwise. Similarly, it can be carried over from Prod to any Archimedean $t$-norm.

We conclude this discussion with a simple illustrative example. Let $(S, \mathscr{F})$ be the $\alpha$-simple space generated by the metric space $(S, d)$ and the distribution function $G$, where $G \in \mathscr{D}^{+}$is continuous and strictly increasing on $[0, \infty)$. Thus, for distinct $p, q \in S$,

$$
F_{p q}(x)=G\left(x / d^{\alpha}(p, q)\right) \text {. }
$$

If $\alpha \leqq 1$ then $d^{\alpha}$ is also a metric on $S$ and $(S, \mathscr{F})$ is the simple space generated by the metric space $\left(S, d^{\alpha}\right)$ and $G$; in this case remark (ii) after Theorem 2.1 applies directly. Suppose therefore that $\alpha>1$. Then, as shown in [19], $(S, \mathscr{F})$ is a Menger space under $\tau_{T}$, where $T$ is strict and multiplicatively generated by

$$
h(x)=\exp \left[-\left(G^{-1}(x)\right)^{1 /(1-\alpha)}\right],
$$

and $G^{-1}$ is the inverse of the restriction of $G$ to $[0, \infty)$. Thus, for distinct $p, q \in S$, 


$$
h F_{p q}(x)= \begin{cases}0, & x \leqq 0 \\ \exp \left[-\left(x /(d(p, q))^{\alpha}\right)^{1 /(1-\alpha)}\right], & x>0\end{cases}
$$

Evaluating the right-hand side of (3.1) yields that, for $z \geqq 0$,

$$
C_{T} F_{p q}(z)=\exp \left[-\alpha(\alpha-1)^{(1-\alpha) / \alpha} z^{1 / \alpha} d(p, q)\right],
$$

whence, substituting in (4.2), we find that

$$
d_{z}(p, q)=\alpha(\alpha-1)^{(1-\alpha) / \alpha} z^{1 / \alpha} d(p, q) .
$$

Thus, for each $z>0, d_{z}$ is a constant multiple of the metric $d$ and consequently, for any distinct points $p, q, r \in S$, we have $M_{z}(p q r)$ if and only if $q$ is between $p$ and $r$ with respect to the metric $d$. It follows that in this case the betweenness relation $M_{z}(p q r)$ is independent of $z$. Furthermore, since it is clear from (4.8) that each $F_{p q}$ is $T$-log-concave, we have, finally, that in an $\alpha$-simple space with $\alpha>1$, the following are equivalent:
(i) $W(p q r)$;
(ii) $E(p q r)$; and
(iii) $q$ is between $p$ and $r$ with respect to the metric $d$.

5. Menger-betweenness. Let $T$ be a $t$-norm and let $\left(S, \mathscr{F}, \tau_{T}\right)$ be a PM space. In [8] Menger postulated that $q$ lies between $p$ and $r$ if these three points are distinct and if, for all $x, y$,

$$
1-F_{p r}(x+y) \geqq T\left(1-F_{p q}(x), 1-F_{q r}(y)\right) .
$$

The probabilistic interpretation of (5.1) is that, for all $x, y$,

$$
\begin{aligned}
& \text { Prob }[\operatorname{dist}(p, r) \geqq x+y] \\
& \quad \geqq T(\operatorname{Prob}[\operatorname{dist}(p, q) \geqq x], \operatorname{Prob}[\operatorname{dist}(q, r) \geqq y]) .
\end{aligned}
$$

The condition (5.1) may be restated in a more perspicacious manner. First of all, let $T^{*}$ be the $t$-conorm of $T$, i.e., the function defined for all $a, b$ in $[0,1]$ by

$$
T^{*}(a, b)=1-T(1-a, 1-b) .
$$

Then (5.1) reads:

$$
F_{p r}(x+y) \leqq T^{*}\left(F_{p q}(x) \cdot F_{q r}(x)\right) .
$$

Next, if $T^{*}$ is continuous then the binary operation $\tau_{T^{*}}$ defined on $\Delta^{+}$ via

$$
\tau_{T^{*}}(F, G)(x)=\inf _{u+v=x} T^{*}(F(u), G(v)),
$$

is a continuous triangle function [17]. Since (5.4) holds for all $x, y$, 
it follows that $q$ is between $p$ and $r$ in Menger's sense-and we write $M(p q r)$-if these three points are distinct and if

$$
F_{p r} \leqq \tau_{T^{*}}\left(F_{p q}, F_{q r}\right) \text {. }
$$

This is the desired reformulation of (5.1). It states that $M(p q r)$ if and only if $\tau_{T^{*}}\left(F_{p q}, F_{q r}\right)$ is an upper bound for $F_{p r}$. Since

$$
\tau_{T}(F, G) \leqq \tau_{T^{*}}(F, G),
$$

for any $F, G \in \Delta^{+}$and any $t$-norm $T$, the condition (5.6) is consistent with the triangle inequality, which states that $\tau_{T}\left(\boldsymbol{F}_{p q}, \boldsymbol{F}_{q r}\right)$ is a lower bound for $F_{p r}$.

It follows at once from (5.7) that $W(p q r)$ implies $M(p q r)$, i.e., that Wald-betweenness implies Menger-betweenness. If $T=$ Min then $T^{*}=\operatorname{Max}$ and a simple calculation shows that $\tau_{\mathrm{Min}}=\tau_{\mathrm{Max}}$. Thus in PM spaces under $\tau_{\mathrm{Min}}$, e.g., in simple spaces the relations $W(p q r)$ and $M(p q r)$ coincide.

In Wald spaces the probabilistic distances between points are generally assumed to be given by independent random variables. Thus, for any three points $p, q, r$, the triangle inequality states that, for all $x$,

$$
\begin{aligned}
F_{p r}(x) & =\operatorname{Prob}[\operatorname{dist}(p, r)<x] \\
& \geqq \operatorname{Prob}[\operatorname{dist}(p, q)+\operatorname{dist}(q, r)<x]=\left(F_{p q} * F_{q r}\right)(x) .
\end{aligned}
$$

Similarly, in this context the analog of (5.2) is

$$
\begin{aligned}
1-F_{p r}(x) & =\operatorname{Prob}[\operatorname{dist}(p, r) \geqq x] \\
& \geqq \operatorname{Prob}[\operatorname{dist}(p, q)+\operatorname{dist}(q, r) \geqq x]=1-\left(F_{p q} * F_{q r}\right)(x),
\end{aligned}
$$

for all $x$, i.e.,

$$
F_{p r} \leqq F_{p q} * F_{q r},
$$

whence, in view of the triangle inequality, $F_{p r}=F_{p q} * F_{q r}$. Thus, in a Wald space, the relations $W(p q r)$ and $M(p q r)$ also coincide.

Generally, however, equality in (5.7) holds only under very restrictive circumstances. It fails, for example, for any $t$-norm $T$ for which $T(a, b)<\operatorname{Min}(a, b)$ for all $a, b \in(0,1)$, and thus for any Archimedean $t$-norm. When this is the case, Menger's betweenness restricts $F_{p r}$ to a certain interval in $\Delta^{+}$. To gain some insight into this situation, we consider several examples.

Let $(S, \mathscr{F})$ be a pseudo-metrically generated PM space (see $\S 2$ ). Since $T_{m}$ is the strongest $t$-norm for this class of spaces and since $T_{m}^{*}(a, b)=\operatorname{Min}(a+b, 1),(5.6)$ becomes

$$
F_{p r}(x) \leqq \inf _{u+v=x} \operatorname{Min}\left(F_{p q}(u)+F_{q r}(v), 1\right)
$$


and we have the following:

Theorem 5.1. If $(S, \mathscr{F})$ is pseudo-metrically generated and if $p, q, r \in S$ are such that $q$ is between $p$ and $r$ for almost all metrics $d \in D$, then $q$ is Menger-between $p$ and $r$, i.e., $M(p q r)$ holds.

Proof. Let $x>0$ be given and choose $u, v \geqq 0$ such that $u+v=x$. If $d(p, r)=d(p, q)+d(q, r)$ and $d(p, r)<x$ then either $d(p, q)<u$ or $d(q, r)<v$. Consequently,

$$
\begin{aligned}
F_{p r}(x) & =\mu\{d \mid d(p, r)<x\} \leqq \mu\{d \mid d(p, q)<u\}+\mu\{d \mid d(q, r)<v\} \\
& =F_{p q}(u)+F_{q r}(v),
\end{aligned}
$$

and the theorem follows.

The converse is false. To see this, consider again the space $(L, \mathscr{F})$, where $L$ is the set of Lebesgue measurable functions on $[0,1]$ and $\mathscr{F}$ is given by (2.15). Let $f(x)=x$ and $h(x)=0$, for $x \in[0,1]$; and let $g(x)$ be given by

$$
g(x)= \begin{cases}1 / 8, & 0 \leqq x \leqq 1 / 8 \\ x, & 1 / 8 \leqq x \leqq 1 / 2 \\ 0, & 1 / 2<x \leqq 1\end{cases}
$$

Since $g(x)>f(x)+h(x)$ for $0 \leqq x<1 / 8, g(x)$ is not between $f(x)$ and $h(x)$ for almost all $x \in[0,1]$. Nevertheless, a straightforward computation shows that $M(f g h)$ holds.

Comparing the above with the known properties of betweenness with respect to the usual $L_{p}$ metrics on $L$, we find that here Mengerbetweenness is strictly weaker than betweenness in any $L_{p}$-metric for $1 \leqq p<\infty$ and not comparable to betweenness in the $L_{\infty}$ metric; and comparing with the results of $\S 2$ shows that Menger-betweenness is a much weaker relation than Wald-betweenness.

As a final example, consider the $\alpha$-simple space generated by $(S, d)$ and the strict distribution function $G$. In this case, using (4.6) and (4.7), some calculation yields that $M(p q r)$ is equivalent to the inequality

$$
d^{\alpha}(p, q) H(u)+d^{\alpha}(q, r) H(v) \leqq d^{\alpha}(p, r) H(u+v), \text { for all } u, v \geqq 0,
$$

where $H$ is the strictly increasing function from $\boldsymbol{R}^{+}$to $\boldsymbol{R}^{+}$given by

$$
H(x)=G^{-1}\left[1-G\left(1 / x^{\alpha-1}\right)\right] .
$$

If we choose $\alpha=2$ and let $G$ be a strict distribution satisfying 
$1-G(x)=G(1 / x)$ (for example, $G(x)=x / 2$ for $0 \leqq x \leqq 1$ and $G(x)=$ $1-1 / 2 x$ for $x \geqq 1$ ) then $H(x)=x$ and (5.10) reduces to

$$
d^{2}(p, q) u+d^{2}(q, r) v \leqq d^{2}(p, r)(u+v), \quad \text { for all } u, v \geqq 0 .
$$

The inequality (5.12) holds if and only if $d(p, r) \geqq \operatorname{Max}(d(p, q), d(q, r))$. In particular, when $(S, d)$ is the Euclidean plane, the set of all points $q$ between two given points $p$ and $r$ is the closed convex region bounded by two circular arcs of radius $d(p, r)$, one with center at $p$, the other with center at $r$. Thus, if $p, q, r$ are vertices of an equilateral triangle then $M(p q r), M(r p q)$ and $M(q r p)$ all hold.

Note that since $H(0)=0$, setting, respectively, $u=0$ and $v=0$ in (5.10) yields that $d(p, r) \geqq \operatorname{Max}(d(p, q), d(q, r))$ is a necessary condition for $M(p q r)$. In our particular example-and indeed, whenever $H(u)+H(v) \leqq H(u+v)$-it is also sufficient.

One might conjecture that, in general, $\{q \mid M(p q r)\}$ is a "convex" set having $p$ and $r$ on its boundary. In any event, Menger-betweenness is a relation which merits further study.

\section{REFERENCES}

1. R. Bellman and W. Karush, On a new functional transform in analysis: the maximum transform, Bull. Amer. Math. Soc., 67 (1961), 501-503.

2. - Mathematical programming and the maximum transform, J. Soc. Indust. Appl. Math., 10 (1962), 550-567.

3. L. M. Blumenthal, Theory and Applications of Distance Geometry, The Clarendon Press, Oxford, 1953.

4. J. B. Brown, Stochastic metrics, Z. Wahrscheinlichkeitstheorie verw. Geb., 24 (1972), 49-62.

5. W. Fenchel, Convex cones, sets, and functions, Lecture notes, Princeton University, 1953.

6. J. F. C. Kingman, Metrics for Wald spaces, J. London Math. Soc., 39 (1964), 129130.

7. C. H. Ling, Representation of associative functions, Publ. Math., Debrecen, 12 (1965), 189-212.

8. K. Menger, Statistical metrics, Proc. Nat. Acad. Sci. U.S.A., 28 (1942), 535-537.

9. R. Moynihan, On the class of $\tau_{T}$ semigroups of probability distribution functions, Aeq. Math., 12 (1975), 249-261.

10. Conjugate transforms for $\tau_{T}$ semigroups, Ph. D. thesis, Univ. of Massachusetts, 1975.

11. - On $\tau_{T}$ semigroups of probability distribution functions II, Aeq. Math., 17 (1978), 19-40.

12. R. Moynihan, B. Schweizer and A. Sklar, Inequalities among operations on probability distributions, General Inequalities I, Proceedings of the First International Conference on General Inequalities held in the Mathematical Research Institute at Oberwolfach, Black Forest, May 10-14, 1976, edited by E. F. Beckenbach, International Series of Numerical Mathematics, Vol. 41, 133-149, Birkhäuser Verlag Basel, 1978.

13. R. Moynihan, Conjugate transforms for $\tau_{T}$ semigroups of probability distribution functions, J. Math. Analysis and Appl., to appear.

14. Conjugate transforms and limit theorems for $\tau_{T}$ semigroups, Studia 
Math., to appear.

15. - Infinite $\tau_{T}$ products of distribution functions, J. Australian Math. Soc., (Series A) 26 (1978), 227-240.

16. F. Rhodes, Convexity in Wald's statistical metric spaces, J. London Math. Soc., 39 (1964), 117-128.

17. B. Schweizer, Multiplications on the space of probability distribution functions, Aeq. Math., 12 (1975), 156-183.

18. B. Schweizer and A. Sklar, Statistical metric spaces, Pacific J. Math., 10 (1960), 313-334.

19. - Triangle inequalities in a class of statistical metric spaces, J. London Math. Soc., 38 (1963), 401-406.

20. B. Schweizer and A. Sklar, Operations on distribution functions not derivable from operations on random variables, Studia Math., 52 (1974), 43-52.

21. B. Schweizer, A. Sklar, and E. Thorp, The metrization of statistical metric spaces, Pacific J. Math., 10 (1960), 673-675.

22. H. Sherwood, On E-spaces and their relation to other classes of probabilistic metric spaces, J. London Math. Soc., 44 (1969), 441-448.

23. - Betweenness in probabilistic metric spaces, Rev. Roum. Math. Pures et Appl., 15 (1970), 1061-1068.

24. D. A. Sibley, A metric for weak convergence of distribution functions, Rocky Mountain J. Math., 1 (1971), 427-430.

25. A. Wald, On a statistical generalization of metric spaces, Proc. Nat. Acad. Sci., U.S.A., 29 (1943), 196-199.

Received October 29, 1975 and in revised form May 17, 1976.

The Mitre Corporation

BEDFORD, MA 01730

AND

UNIVERSITY OF MASSACHUSETTS

AMHERST, MA 01003 


\section{PACIFIC JOURNAL OF MATHEMATICS}

EDITORS

DONALD BABBITT (Managing Editor)

University of California

Los Angeles, California 90024

HUGo RossI

University of Utah

Salt Lake City, UT 84112

C. C. MOORE

J. DUGUNDJI

Department of Mathematics

University of Southern California

Los Angeles, California 90007

R. FinN AND J. MiLgraM

Stanford University

Stanford, California 94305

University of California

Berkeley, CA 94720

\section{ASSOCIATE EDITORS}

E. F. BrCKENBACH

B. H. NeUmanN

F. WOLF

K. YoshidA

\section{SUPPORTING INSTITUTIONS}

UNIVERSITY OF BRITISH COLUMBIA

UNIVERSITY OF SOUTHERN CALIFORNIA

CALIFORNIA INSTITUTE OF TECHNOLOGY

STANFORD UNIVERSITY

UNIVERSITY OF CALIFORNIA

UNIVERSITY OF HAWAII

MONTANA STATE UNIVERSITY

UNIVERSITY OF TOKYO

UNIVERSITY OF NEVADA, RENO

UNIVERSITY OF UTAH

NEW MEXICO STATE UNIVERSITY

WASHINGTON STATE UNIVERSITY

OREGON STATE UNIVERSITY

UNIVERSITY OF WASHINGTON

UNIVERSITY OF OREGON 


\section{Pacific Journal of Mathematics}

\section{Vol. 81, No. $1 \quad$ November, 1979}

Thomas E. Armstrong, Simplicial subdivision of infinite-dimensional compact cubes ..................................... 1

Herbert Stanley Bear, Jr., Approximate identities and pointwise convergence ................................

Richard David Bourgin, Partial orderings for integral representations on convex sets with the Radon-Nikodým property..................

Alan Day, Herbert S. Gaskill and Werner Poguntke, Distributive lattices

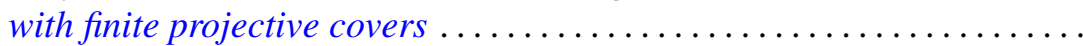

Heneri Amos Murima Dzinotyiweyi and Gerard L. G. Sleijpen, A note on measures on foundation semigroups with weakly compact orbits ......

Ronald James Evans, Resolution of sign ambiguities in Jacobi and Jacobsthal sums ...................................

John Albert Fridy, Tauberian theorems via block dominated matrices ......

Matthew Gould and Helen H. James, Automorphism groups retracting onto

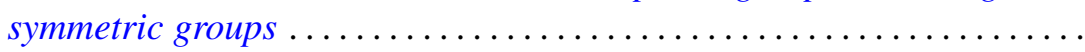

Kurt Kreith, Nonlinear differential equations with monotone solutions . . . . 101

Brian William McEnnis, Shifts on indefinite inner product spaces........ 113

Joseph B. Miles, On entire functions of infinite order with radially

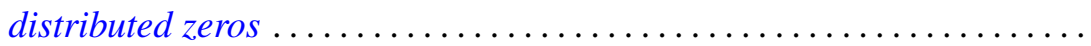

Janet E. Mills, The idempotents of a class of 0-simple inverse semigroups ...............................

Edward Jean Moulis, Jr., Generalizations of the Robertson functions ...

Richard A. Moynihan and Berthold Schweizer, Betweenness relations in probabilistic metric spaces.......................

Stanley Ocken, Perturbing embeddings in codimension two ....

Masilamani Sambandham, On the average number of real zeros of a class of random algebraic curves.

Jerry Searcy and B. Andreas Troesch, A cyclic inequality and a related eigenvalue problem.

Roger R. Smith and Joseph Dinneen Ward, $M$-ideals in $B\left(l_{p}\right)$...

Michel Talagrand, Deux généralisations d'un théorème de I. Namioka ..

Jürgen Voigt, $O n Y$-closed subspaces of $X$, for Banach spaces $X \subset Y$;

existence of alternating elements in subspaces of $C(J)$

Sidney Martin Webster, On mapping an $n$-ball into an $(n+1)$-ball in complex spaces

David J. Winter, Triangulable subalgebras of Lie p-algebras ... 